University of Nebraska - Lincoln

DigitalCommons@University of Nebraska - Lincoln

CSE Conference and Workshop Papers

Computer Science and Engineering, Department of

2012

\title{
A Preliminary Model for Comfortable Approach Distance based on Environmental Conditions and Personal Factors
}

Brittany Duncan

University of Nebraska-Lincoln, bduncan@unl.edu

Robin R. Murphy

Texas A\&M University, murphy@cse.tamu.edu

Follow this and additional works at: http://digitalcommons.unl.edu/cseconfwork

Duncan, Brittany and Murphy, Robin R., "A Preliminary Model for Comfortable Approach Distance based on Environmental

Conditions and Personal Factors" (2012). CSE Conference and Workshop Papers. 246.

http://digitalcommons.unl.edu/cseconfwork/246

This Article is brought to you for free and open access by the Computer Science and Engineering, Department of at DigitalCommons@University of Nebraska - Lincoln. It has been accepted for inclusion in CSE Conference and Workshop Papers by an authorized administrator of

DigitalCommons@University of Nebraska - Lincoln. 


\title{
A Preliminary Model for Comfortable Approach Distance based on Environmental Conditions and Personal Factors
}

\author{
Brittany A. Duncan and Robin R. Murphy \\ Department of Computer Science and Engineering \\ Texas A\&M University \\ College Station, TX 77843-3112 \\ Email: \{bduncan, murphy\}@cse.tamu.edu
}

\section{POSTER PAPER}

\begin{abstract}
This paper presents a model of "comfortable distance" that captures the factors and conditions known to affect personal space in human-human and human-robot interactions, as well as any identified relationships between them. In the first known human-robot interaction (HRI) survey on approach distance, 19 papers were reviewed and the "comfortable distance" model was synthesized with three distinct types of inputs: environmental conditions, personal factors, and agent factors. Five environmental conditions (lighting, ceiling height, indoor/outdoor, room size, and barrier height) and seven personal factors (gender, age, mood, personality, pet ownership, robot experience, and sitting/standing) structure the model, with four agent factors (angle of approach, height of agent, speed of approach, and gaze) used as tuning parameters to produce behaviors with appropriate distances. Currently, HRI researchers generally focus on one factor at a time (e.g., approach angle or approach speed), without considering the previous work in adjacent fields, such as psychology and other social sciences. This has resulted in environmental factors being ignored by the HRI community. The "comfortable distance" model is a new tool for HRI researchers and is expandable so that it can incorporate new factors as they are identified. This survey will inform researchers about factors which had been previously overlooked in the field of HRI and will allow future researchers to consider the impact of identified variables to create more complete experiments.
\end{abstract}

Keywords-Interaction Control in Robotic Systems; Human Factors and Evaluation Methodologies.

\section{INTRODUCTION}

The purpose of this literature review is to examine the relevant works from psychology and human-robot interaction in order to determine the factors that might affect a general model of comfortable approach distance. Papers were included in this review only if they are directly applicable to approach distance specifically, rather than proxemics in general. The result of this review is the creation of a preliminary a model for comfortable approach distance (see Figure 1). This work is based on the CASA (computers are social actors) model

This material is based upon work supported by the National Science Foundation Graduate Research Fellowship.

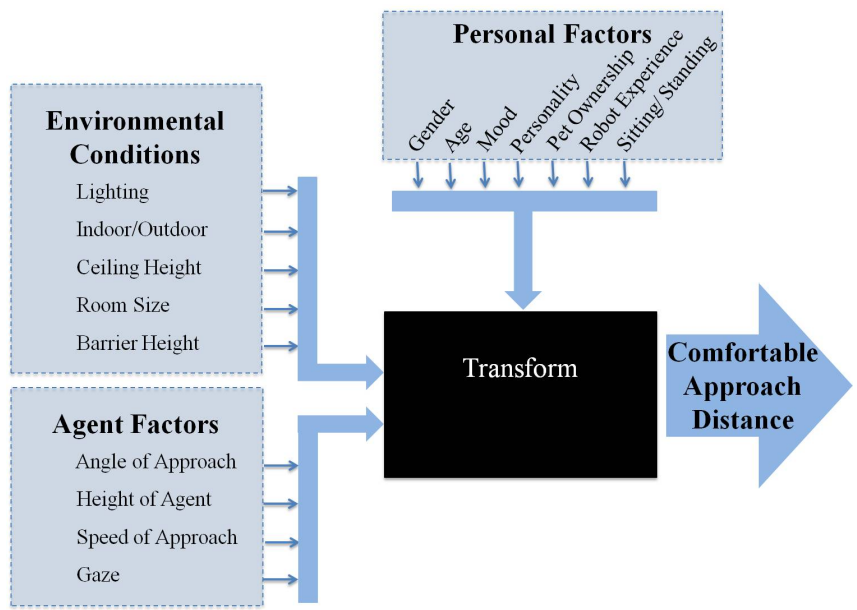

Figure 1. "Comfortable Distance" Model, incorporating the environmental conditions, agent factors, and personal factors identified from the literature. The transform black box represents the interactions between the factors, which can be partially gathered from the literature. The output is the distance that a human would feel comfortable being distanced from a robot or another human.

by Nass, Steuer, and Tauber [1], which considers human studies to be directly applicable to human-computer and human-robot interactions; therefore the rest of the paper will present both sets of findings together. Psychologists have studied environmental conditions extensively, but HRI researchers have not examined these conditions. Agent and personal factors have been examined by both psychologists and HRI researchers. It is important to isolate the factors by environment, agent, and human so that experimenters know the conditions they will try to isolate in an experiment versus the conditions they can change. As HRI experiments continue in this area, the model can be expanded in order to include new findings. 
Researchers in psychology and HRI have looked at 16 factors or conditions in three areas: environment, personal (to the subject), and agent (experimenter or robot). Five of these conditions belong to the environment and include: lighting, ceiling height, location (indoor/outdoor), room size, and barrier height. Seven factors are included in the personal (to the subject) group: gender, age, mood, personality, pet ownership, robot experience, and position (sitting/standing). The final four factors belong to the agent, the experimenter or robot: angle of approach, height of agent, speed of approach, and gaze.

The application of the "comfortable distance" model is important to HRI for three reasons: to avoid duplication of efforts, to create experiments that control for the appropriate factors, and to inform the design of personal and service robots in the future. By having a model with this information already incorporated, researchers will be able to readily identify areas of research that have been examined. In order to run experiments that produce generalizable results, scientists need to have an overview of the factors that might impact their experiments so they can adequately control for them. To this end, it is important to know what personal factors should be collected during the course of the experiment and the environmental conditions that should be reported with the results. With all of the information collected into a model, designers of personal and service robots will be able to incorporate any a priori information they may have to produce results that humans would find comfortable.

The remainder of this article is organized as follows. Section II will discuss the research into environmental conditions and the comfort measures used in these studies. Section III will discuss the personal factors and associated measures. Section IV will present the relevant agent factors and how they have been measured. Section V will present the inputs for the model. Section VI will present the findings. Finally, Section VII will present the conclusions and future work based on the information from throughout this paper. This work is expected to contribute: i) a model for comfortable approach distance and ii) areas which merit future research.

\section{ENVIRONMENTAL CONDITIONS}

Environmental conditions are those features of the environment that are readily identifiable and can be measured. The conditions that have been studied include: lighting, ceiling height, room size, barrier height, and location (indoor/outdoor).

Adams and Zuckerman [2] studied how lighting and room size impact personal space between for human-human interactions. Their findings demonstrated that a reduction in lighting has a similar effect on interpersonal space as a decrease in room size.

Cochran, Hale, and Hassim [3] studied how indoor and outdoor locations impact personal space for human-human interactions. The indoor space had a significantly larger interpersonal distance than the outdoor space. In this study, the ceiling height was unbounded in the outdoor condition.

Cochran and Urbanczyk [4] studied how different ceiling height conditions impact personal space for human-human interactions. This study determined that a lower ceilinged room resulted in a larger interpersonal distance being required.

Meyers-Levy and Zhu [5] studied how variations in ceiling height can prime concepts of freedom versus confinement. Chinese lanterns were used to enhance participants' attentiveness to the ceiling height. This study determined that a high ceiling activated freedom-related concepts.

Marcilly and Luyat [6] examined how eye height affects judgment of passage under a barrier. This was tested using glasses to change effective eye height. Effective eye height is defined as the "projection of the observer's eye height into the environment." This study showed that if a barrier is less than 1.1 times the effective eye height, then individuals perceive that they cannot pass under it without changing their posture.

In the five identified studies of environmental conditions on proxemics, five conditions were covered: lighting, ceiling height, room size, barrier height, and location (indoor/outdoor). Lighting was examined only by Adams and Zuckerman [2]. Ceiling height was covered by Cochran, Hale, and Hassim [3], Cochran and Urbanczyk [4], and MeyersLevy and Zhu [5]. Room size was studied only by Adams and Zuckerman [2]. Barrier height was investigated only by Marcilly and Luyat [6]. Finally, location was researched only by Cochran, Hale, and Hassim [3].

\section{PERSONAL FACTORS}

Personal factors are those features of the human that are identifiable and whose impact can be measured. The factors that have been studied include: gender, age, mood, personality, pet ownership, robot experience, and position (sitting/standing).

Kinzel [7] studied the impact of the angle of approach on interpersonal distance in violent prisoners for humanhuman interactions. This was the first study to use the stop distance technique and was the only study found to take mood into account. This study showed that the average rear personal zone was larger for violent prisoners and that the average front zone was larger for non-violent prisoners. The total zone was over four times larger for violent prisoners.

Mumm and Mutlu [8] examined the four models of interpersonal distancing (Reciprocity, Compensation, Attraction-Mediation, and Attraction-Transformation) for human-robot interactions. To do this, they studied the impacts of pet ownership, and gender on human-robot distancing with 
the Wakamaru robot. This study found that males distanced themselves further than females and pet owners distanced themselves further than non-pet owners.

Takayama and Pantofaru [9] studied human-robot distancing based on pet ownership and robot experience. This study showed that pet owners and people with at least a year of robot experience maintain a smaller distance from the PR2 robot and that gaze combined with gender has a significant impact on distance.

Syrdal, Dautenhahn, et al [10] examined the effects of subject personality and the impact of the position of the subject, sitting or standing, on the preferred approach direction of the Peoplebot robot. The effects in this study were too small to be considered significant for the sample size used in the experiments, but suggest that higher extraversion scores led to a better tolerance of inappropriate robot behavior.

Syrdal, Koay, et al [11] studied the role of individual differences on spatial preferences, but focused on the impact of personality on human-robot distancing with the Peoplebot robot and the impact of gender on approach angle. As part of this study, the authors identified a set of external (cultural norms, situational/interactional context, degree of aquaintance between actors, and relative social status between actors) and internal factors (gender, personality, physical attributes, health and medical factors, and other individual differences). This study showed that gender impacts approach angle preference, and that extraversion and conscientiousness impact distance.

Walters, Dautenhahn, et al [12] studied the impact of personality on human-robot distancing with the Peoplebot robot. This study showed that proactive subjects allowed the robot to approach closer.

Hartnett [13] studied how interpersonal distances in humanhuman interactions changed based on whether the participants were approaching someone who was sitting versus standing, whether standing height had any effect on this distance, and the impact of gender. Males and females both maintained twice as much distance from a tall person than a short person. Females approached more closely to someone who was sitting and males approached more closely to someone who was standing.

Pacchierotti, Christensen, and Jensfelt [14] studied hallway passing behaviors for human-robot interactions with the Peoplebot using robotics students as subjects with the goal of embodying "social intelligence". This study was used to test parameters of the robot and found that users preferred a robot to move fast (between 0.25 to 0.39 meters per second), but this might be explained by the participants' comfort level with the robot. Another finding is that there were two types of users: those who treated the robot as a person and those who treated the robot as a machine.
Walters, Koay, et al [15] studied the impact of personality on likeability of a tall or short and humanoid or mechanoid Peoplebot robot for human-robot interactions. The only significant effects were related to the stated preferences and the interaction distances. There was only a 0.2 meter difference between the tall and short condition.

Oosterhout and Visser [16] studied the impact of person age on likeability and approach distance for human-robot interactions with Mobi, Sr. and Mobi, Jr. Children were 3.5 times more likely to interact with the short robot, while young adults were 7 times more likely and adults are 2.8 times more likely to interact with the taller robot. There was a 0.63 meter difference between the two robots.

Butler and Agah [17] studied the impact of technical experience (classified as robot experience) and gender on the comfortable approach distance in three types of interactions (approaching, avoiding, and non-interactive) for human-robot interactions with the Nomadic Scout II robot. An effect was found based on technical background, but no impact was found based on gender. None of the results were analyzed for statistical significance.

In the eleven identified studies of personal factors on comfortable approach distance, seven factors were covered: gender, age, mood, personality, pet ownership, robot experience, and position (sitting/standing). Gender was studied by Mumm and Mutlu [8], Takayama and Pantofaru [9], Syrdal, Koay, et al [11], Hartnett [13], and Butler and Agah [17]. Age was examined only by Oosterhout and Visser [16]. Mood was covered only by Kinzel [7]. Personality was researched by Takayama and Pantofaru [9], Syrdal, Dautenhahn, et al [10], Syrdal, Koay, et al [11], Walters, Dautenhahn, et al [12], and Walters, Koay, et al [15]. Pet ownership was analyzed by Mumm and Mutlu [8], Takayama and Pantofaru [9], and Butler and Agah [17]. Robot experience was studied by Takayama and Pantofaru [9], Pacchierotti, Christensen, and Jensfelt [14], and Butler and Agah [17]. Finally, position was covered by Syrdal, Dautenhahn, et al [10] and Hartnett [13].

\section{AGENT FACTORS}

Agent factors are those features of the agent that can be changed based on the situation. The factors that have been studied include: angle of approach, height of agent, speed of approach, and gaze.

Adams and Zuckerman [2] studied the impact of the angle of approach on interpersonal distance for human-human interactions. Only females were included in this study, and the experimenter who approached them was also a female. Their study showed that as the direction of approach moved from front to rear, the distance requirements increased. 
Kinzel [7] studied the effect the angle of approach on interpersonal distance in violent prisoners for human-human interactions. This study showed that the average rear personal zone was larger for violent prisoners and that the average front zone was larger for non-violent prisoners.

Caplan and Goldman [18] studied personal space violations as a function of height in human-human interactions. This study showed that people were more likely to invade the personal space of a short person over a tall person, regardless of gender.

Hartnett [13] studied how interpersonal distances changed based on whether the participants were approaching someone who was sitting versus standing, and whether standing height had any effect on this distance in human-human interactions. People maintained twice as much distance from a tall person than a short person. Females approached more closely to someone who was sitting and males approached more closely to someone who was standing.

Mumm and Mutlu [8] examined the four models of interpersonal distancing (Reciprocity, Compensation, Attraction-Mediation, and Attraction-Transformation). To do this, they studied the impacts of gaze behavior on humanrobot distancing with the Wakamaru robot. This study found that as gaze increased, so did the distance between the human and the robot.

Takayama and Pantofaru [9] studied human-robot distancing based on gaze behaviors with the PR2 robot. This study showed that gaze combined with gender has a significant impact on distance.

Pacchierotti, Christensen, and Jensfelt [14] studied hallway passing behaviors using robotics students as subjects with the goal of embodying "social intelligence" for human-robot interactions with the Peoplebot robot. This study was used to test parameters of the robot and found that users preferred a robot to move fast (between 0.25 to 0.39 meters per second).

Syrdal, Dautenhahn, et al [10] studied the impact of the position of the subject, sitting or standing, on the preferred approach direction of the Peoplebot robot for human-robot interactions. The effects in this study were too small to be considered significant for the sample size used in the experiments, but suggest that subjects preferred to be approached from the front, but not directly (i.e. either front-right or front-left).

Walters, Koay, et al [15] studied the impact of height and design on approach direction and likeability of a tall or short and humanoid or mechanoid robot for human-robot interaction with the Peoplebot robot. The only significant effects were related to the stated preferences and the interaction distances. There was only a 0.2 meter difference between the tall and short condition.
Butler and Agah [17] studied the impact of robot speed and robot body design (including height) on the comfortable approach distance in three types of interactions (approaching, avoiding, and non-interactive) for human-robot interaction with the Nomadic Scout II robot. The addition of the humanoid body attachment (which created a robot that was 1.7 meters tall) created negative results in "almost all cases". The shorter robot was only 0.35 meters tall. The comfortable speeds were identified as 0.254 and 0.381 meters per second.

Oosterhout and Visser [16] studied the impact of height on likeability and approach distance for human-robot interaction with Mobi, Sr. and Mobi, Jr. Children were 3.5 times more likely to interact with the short robot, while young adults were 7 times more likely and adults are 2.8 times more likely to interact with the taller robot. There was a 0.63 meter difference between the two robots.

In the twelve identified studies of agent factors on comfortable approach distance, four factors were covered: angle of approach, height of agent, speed of approach, and gaze. Angle of approach was covered by Adams and Zuckerman [2], Kinzel [7], Walters, Koay, et al [15], and Syrdal, Dautenhahn, et al [10]. Height of agent was studied by Caplan and Goldman [18], Hartnett [13], Butler and Agah [17], Walters, Koay, et al [15], and Oosterhout and Visser [16]. Speed of approach was tested by Pacchierotti, Christensen, and Jensfelt [14] and Butler and Agah [17]. Finally, gaze was examined by Mumm and Mutlu [8] as well as Takayama and Pantofaru [9].

\section{MODEL INPUTS}

This section will summarize the central finding for each of the three areas of model input covered in this paper, including: environmental conditions, personal factors, and agent factors. Each of these areas has two or more supporting facts, which will be summarized from the studies presented earlier.

\section{A. Environmental Conditions}

Room size is centrally related to all environmental condition findings.

1) Room size is inversely related to personal space.

2) Lighting is directly related to room size.

3) Ceiling height is directly related to room size and outdoor locations are considered to have a ceiling of infinite height.

\section{B. Personal Factors}

Personal factors were varied and sometimes conflicting based on their reporting in the literature.

1) The main gender finding was that males distanced themselves further than women from robots.

2) Age was only examined in one study with the participants grouped into children, young adult, and adult. Young adults showed the most sensitivity to robot height and 
were 7 times more likely to interact with a tall robot than a short robot.

3) Mood has only been studied with regard to violent prisoners, and this study showed that violent prisoners had a total personal space zone that was four times larger than other prisoners.

4) Different personality dimensions were explored, and it was suggested that: extraverts had a better tolerance of inappropriate robot behavior and proactive subjects allowed the robot to approach closer.

5) Pet ownership findings were conflicting, with one study suggesting that pet owners distance themselves further from a robot, and the other suggesting the opposite.

6) Experience with robots led to users who maintained a smaller distance from the robot and preferred a faster moving robot.

\section{Agent Factors}

Agent factors have not been very well studied in the literature.

1) Angle of approach was found to increase comfortable approach distance as the angle moved from front to back.

2) Height had an effect in that the taller an agent is, the less likely people are to invade its space.

3) The suggested approach speed for comfortable distance trials is approximately 0.15 to 0.20 meters per second to minimize measurement error.

4) An increase in gaze usually results in a larger personal space being necessary.

\section{FINDINGS}

This review of literature has not only produced a preliminary model of "comfortable distance" based on relationships found in both psychology and HRI studies, but has found areas where the literature is incomplete. Out of the four agent factors that were identified, only two papers covered more than one factor, and these only covered two factors [15], [17]. From the seven identified personal factors, the paper that covered the most factors only covered four of them [9]. Another paper covered three factors [17], with four papers covering two of these factors [8], [10], [11], [13]. Since the agent factors and personal factors have not all been studied under the same conditions, nor in the same set of experiments, it is difficult to anticipate what interactions might occur. HRI studies are incomplete in their study of the environmental conditions, which can be generalized from psychology, but have not been examined for their impact on the current studies.

Proxemic interactions have been a burgeoning area in HRI, and the subfield of comfortable approach distance has been no exception, but in order to create a more complete picture of the factors that affect these interactions a model should be created. A preliminary version of this model has been presented here, with suggested inputs presented in the previous section. In order to complete this model, HRI studies should be completed that take all factors in a given area into account to determine interactions.

\section{CONCLUSIONS AND FUTURE WORK}

This paper is a synthesis of work from human-human and human-robot studies into the "comfortable distance" model for interactions. This model incorporates 16 factors or conditions identified from 19 papers into three types of factors or conditions: environmental, personal, and agent-controlled. The most significant relationship is that environmental conditions are centrally related to room size, where ceiling height and lighting are directly related to room size.

While many factors have been identified in this paper, several also require further study. Environmental conditions (excluding ceiling height), age, and mood were the least studied factors with only 1 study each. Gaze has been studied by two researchers, but they ended up with conflicting results. In order to better generalize results into a model, such as the one suggested in this paper, experiments will need to be replicated. HRI studies should be completed that take all factors in a given area into account to determine interactions.

\section{REFERENCES}

[1] C. Nass, J. Steuer, and E. R. Tauber, "Computers are Social Actors," Proceedings of the ACM CHI 94 Human Factors in Computing Systems Conference, Boston, Massachusetts, 1994.

[2] L. Adams and D. Zuckerman, "The Effect of Lighting Conditions on Personal Space Requirements," Journal of General Psychology, vol. 118, pp. 335-340, 1991.

[3] C. D. Cochran, et al, "PERSONAL SPACE REQUIREMENTS IN INDOOR VERSES OUTDOOR LOCATIONS,” Journal of Psychology, vol. 117, p. 121, 1984.

[4] C. D. Cochran and S. Urbanczyk, "The Effect of Availability of Vertical Space on Personal Space," Journal of Psychology, vol. 111, pp. 137-140, 1982.

[5] J. Meyers-Levy and R. J. Zhu, "The Influence of Ceiling Height: The Effect of Priming on the Type of Processing that People Use," Journal of Consumer Research, vol. 34, pp. 174-186, 2007.

[6] R. Marcilly and M. Luyat, "THE ROLE OF EYE HEIGHT IN JUDGMENT OF AN AFFORDANCE OF PASSAGE UNDER A BARRIER,' Current Psychology Letters, vol. 24, pp. 12-24, 2008.

[7] A. F. KINZEL, "Body-Buffer Zone in Violent Prisoners," Am J Psychiatry, vol. 127, pp. 59-64, July 1, 19701970.

[8] J. Mumm and B. Mutlu, "Human-robot proxemics: physical and psychological distancing in human-robot interaction," presented at the Proceedings of the 6th international conference on Human-robot interaction, Lausanne, Switzerland, 2011.

[9] L. Takayama and C. Pantofaru, "Influences on proxemic behaviors in human-robot interaction," in Intelligent Robots and Systems, 2009. IROS 2009. IEEE/RSJ International Conference on, 2009, pp. 5495-5502.

[10] D. S. Syrdal, et al, “'Doing the right thing wrong' - Personality and tolerance to uncomfortable robot approaches," in Robot and Human Interactive Communication, 2006. ROMAN 2006. The 15th IEEE International Symposium on, 2006, pp. 183-188.

[11] D. S. Syrdal, et al, "A personalized robot companion? - The role of individual differences on spatial preferences in HRI scenarios," in Robot and Human interactive Communication, 2007. RO-MAN 2007. The 16th IEEE International Symposium on, 2007, pp. 1143-1148.

[12] M. L. Walters, et al, "The influence of subjects' personality traits on personal spatial zones in a human-robot interaction experiment," in Robot and Human Interactive Communication, 2005. ROMAN 2005. IEEE International Workshop on, 2005, pp. 347-352.

[13] J. J. Hartnett, "Body height, position, and sex as determinants of personal space," Journal of Psychology, vol. 87, p. 129, 1974. 
[14] E. Pacchierotti, et al, "Human-robot embodied interaction in hallway settings: a pilot user study," in Robot and Human Interactive Communication, 2005. ROMAN 2005. IEEE International Workshop on, 2005, pp. 164-171.

[15] M. L. Walters, et al, "Preferences and perceptions of robot appearance and embodiment in human-robot interaction trials," in Artificial Intelligence and Simulation of Behaviour (AISB), Edinburgh, Scotland, 2009, pp. 136-143.

[16] T. V. Oosterhout and A. Visser, "A Visual Method for Robot Proxemics Measurements," in Proc. of Metrics for Human-Robot Interaction, a Workshop at ACM/IEEE HRI 2008, Amsterdam, the Netherlands, 2008, pp. 61-68.

[17] J. T. Butler and A. Agah, "Psychological Effects of Behavior Patterns of a Mobile Personal Robot," in Autonomous Robots, Springer Netherlands, 2001, 10(2), pp. 185-202.

[18] M. E. Caplan and M. Goldman, "PERSONAL SPACE VIOLATIONS AS A FUNCTION OF HEIGHT,' Journal of Social Psychology, vol. 114, p. $167,1981$. 